ourselves with the numbers of empty plates, of plates containing no rice but some (that is, at least one scoop of) beans, of plates containing no beans but some rice, and of plates containing some of each. These numbers are $a, b, c$ and $d$ of our contingency table, and we use the marginal totals to compute the expected numbers of each of the four kinds of plates. The numbers of scoops of beans and of rice on plates having at least some of either are no more necessary to this test of association than are the severity of cancer or the number of packs smoked per day to a test of association between smoking and lung cancer.

MATTHEW H. GREENSTONE
Department of Entomology
and Nematology,
University of Florida,
Gainesville, Florida 32611, USA

1. Greenstone, M. H. Nature 282, 501-503 (1979).

\section{Plasmoid confinement by the charged particle micro-fields}

DIJKHUIS $^{1}$ has recently proposed a 'boson model' which explains ball lightning in terms of electric charge. It is based on the hypothesis that turbulence in the ball lightning plasma enhances charge transfer to the point that charged vortices transport electricity the way electrons do in a superconductor. The result is described as a sponge-like structure of tenuous vortex cores nested in dense ambient plasma. By reasonings based on Landau damping and local deviations from charge neutrality it is argued that the size of the plasma vortices behaving as charged bosons apparently lies around 1 Debye length.

Plasmoid models of ball lightning are normally taken to be ruled out by the plasma virial theorem yielding the result, at least in its usual continuum formulation, that a plasmoid-defined as a selfcontained bundle of electromagnetic and material energy-cannot exist. The theorem states that the plasmoid energy contents-electromagnetic as well as gas kinetic-are all positive and together drive expansion. However, the boson model for plasma turbulence is claimed to lift the virial constraint on maximum energy content with negative potential energy from interaction between plasma vortices.

The negative interaction energy necessary for the boson model conflicts with the positive-definite nature of all the energies in the fluid virial theorem. Note that the proposed boson model specifically involves interactions and structures on the plasma microfield scale that is, the Debye length. As discussed by Gerjoy and Stabler ${ }^{2}$ the averaged, 'smoothed-out', plasma descriptior inherent in the fluid virial theorem may, in some respects, be misleading. Resort has then to be taken in a perfectly general and purely particle system formulation of the virial theorem. The electrostatic energy is then given by the double sum

$$
W_{\mathrm{el}}=\frac{1}{4 \pi \varepsilon_{0}} \sum_{i<k} e_{i} e_{k} r_{i k}^{-1} ; r_{i k}=\left|\mathbf{r}_{i}-\mathbf{r}_{k}\right|
$$

Note that a slight excess of negative charges around each positive charge will make $W_{\text {el }}$ go negative ${ }^{2}$. However, this is also that condition which will distribute the source charges $e_{k}$ so as to minimize the electrostatic potential $\phi^{i}$ at the field point $i$ as well as to make the related field strength $\mathbf{E}^{i}=-\nabla \phi^{i}$ small

$$
\phi^{i}=\frac{1}{4 \pi \varepsilon_{0}} \sum_{k \neq i} e_{k} r_{i k}^{-13}
$$

The influence of a negative contribution $W_{\text {el }}$, as indicated above, to a virial theorem reasoning has been discussed and estimated in ref. 2. For a plasma with thermal energy like that assumed for the boson model it has been proved to be insignificant, thus validating the results of simpler fluid virial theorem reasoning.

In a similar way, the magnetic energy $W_{\mathrm{m}}$ and vector potential $\mathbf{A}^{i}$ can be expressed in terms of the microscopic currents $\mathbf{j}_{i}$ and $\mathbf{j}_{k}$ carried by the charges $e_{i}$ and $e_{k}$

$$
W_{\mathrm{m}}=\frac{\mu_{0}}{4 \pi} \sum_{i<k} \mathbf{j}_{i} \cdot \mathbf{j}_{k} r_{i k}^{-1}, \mathbf{A}^{i}=\frac{\mu_{0}}{4 \pi} \sum_{i \neq k} \mathbf{j}_{k} r_{i k}^{-1}
$$

Here it is noted that a hypothetical current distribution where adjacent current elements are directed oppositely would make $W_{\mathrm{m}}$ go negative but, also, make $\mathbf{A}^{i}$ and $\mathbf{B}^{i}=$ curl $\mathbf{A}^{i}$ attain vanishingly small values. Contrary to the claim in ref. 1 , there seems to be no way by which the magnetic interaction energy can attain a large enough negative value to affect significantly the expansion-driving thermal effects. Essentially the same result, in conflict with the proposed boson model for ball lightning, was also obtained by Liboff and $\mathrm{Lie}^{3}$ who used instead the quasi-relativistic Coulomb gauge expression for $\mathbf{A}^{i}$ in their derivation of a general virial theorem in a particle formulation.

I conclude that the various formulations of the virial theorem as discussed above are all based on usual charged-particle electrodynamics where fields and forces follow from positions and velocities. The power then of the theorem to deny plasmoid self-confinement lends support to that theory ${ }^{4}$ where the confinement is provided by the radiation fields associated with particle acceleration.

\section{E. A. Witalis Research Institute of National Defense, Stockholm, Sweden}

1. Dijkhuis, G. C. Nature 284, 150 (1980). 2. Gerjoy, S. \& Stabler, R. C. Phys. Fluids 7, 920 (1964) 3. Liboff, R. L. \& Lie, T.-J. Phys. Fluids 11, 1943 (1968). 4. Bergström, A. Phys. Rev. D 8, 4394 (1973).
DIJKHUIS REPLIES--It is a delusion to claim perfect generality for the purely particle system formulation of the virial theorem. Particles in a plasma obey classical equations of motion as long as electromagnetic fields do not change appreciably over their dimension. This classical limit is certainly appropriate for any stable plasma in a smooth electromagnetic field. However, in a turbulent plasma electromagnetic fields may have strong local fluctuations. In our approach to ball lightning, turbulent fluctuations of local electromagnetic fields become so violent that the motion of particles must be treated quantum mechanically.

A particle with mass $m$ and charge $q$, moving with velocity $\mathbf{v}$ in an electromagnetic field with vector potential $\mathbf{A}$ and electrostatic potential $\phi$, has canonical momentum $\mathbf{p}=m \mathbf{v}+q \mathbf{A}$ and hamiltonian $H=(\mathbf{p}-q \mathbf{A})^{2} / 2 m+q \phi$. In our quantummechanical context, $p=-i \hbar \nabla$ is an operator which does not commute with arbitrary functions of position vector $\mathbf{r}$. Thus $\frac{1}{2} q(\mathbf{v} \times \mathbf{B}-\mathbf{B} \times \mathbf{v})$ (ref. 1) denotes the Lorentz force on a wave packet moving in a magnetic field $\mathbf{B}=\boldsymbol{\nabla} \times \mathbf{A}$. In the absence of magnetic fields a wave packet has $\mathbf{p}=$ $m \mathbf{v}, \quad H=\frac{1}{2} m v^{2}+q \phi$, and the virial theorem reads $\mathrm{d} / \mathrm{d} t\langle\mathbf{r} \cdot m \mathbf{v}\rangle=m\left\langle v^{2}\right\rangle-$ $q\langle\mathbf{r} \cdot \boldsymbol{\nabla} \phi\rangle$ (ref. 1), where the brackets denote expectation values with wave functions from the energy representation. Inclusion of magnetic interaction between wave packets leads after lengthy commutator bracket calculations to a quantum-mechanical virial theorem of the form

$$
\begin{aligned}
\frac{\mathrm{d}}{\mathrm{d} t}\langle\mathbf{r} \cdot m \mathbf{v}\rangle= & m\left\langle v^{2}\right\rangle+\frac{1}{2} q\langle\mathbf{r} \times \mathbf{v} \cdot \mathbf{B}+\mathbf{B} \cdot \mathbf{r} \times \mathbf{v}\rangle \\
& -q\langle\mathbf{r} \cdot \boldsymbol{\nabla} \phi\rangle
\end{aligned}
$$

where for a stable plasmoid at least one term on the right has to be negative. With $\rho^{1 / 2} e^{\mathrm{i} \theta}$ as wave function for a boson state with charge density $\rho$ and phase $\theta$ (ref. 2), expectation values in equation (1) include the quantum-mechanical term $-\hbar^{2} / 2 m$ $\left(\nabla^{2} \rho^{1 / 2}\right) / \rho^{1 / 2}$, from which a spherically symmetric solution with negative energy was derived in our letter ${ }^{3}$. In the classical limit $\hbar \rightarrow 0$, expectation values can be dropped and all quantities commute, so that the usual terms for kinetic, magnetic and electric energy are recovered.

I maintain that (1) the quantummechanical version of the virial theorem admits electromagnetic self-confinement of a plasma, and (2) strong plasma turbulence leads to a self-confined boson state in ball lightning.

\section{G. C. Diskhuis}

Zeldenrust College, Terneuzen, The Netherlands

Schiff L I Ouanum Mechanics 3rd edn - 177-180 (McGraw-Hill, New York, 19681.

2. Feynman, R. P., Leighton, R. B.\& Sands, M. The Feynman Lectures on Physics Vol. III, Section 21 iAddisonWesley, New York, 1966).

3. Dijkhuis, G.C. Nature 284, $150 ; 1980)$. 